\title{
Effects of boundaries on structure formation in low-dimensional colloid model systems near the liquid-solid-transition in equilibrium and in external fields and under shear
}

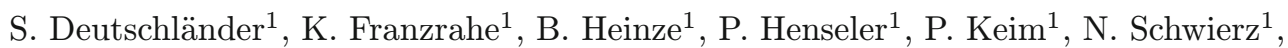
U. Siems ${ }^{1}$, P. Virnau ${ }^{2}$, D. Wilms ${ }^{2,3}$, K. Binder ${ }^{2}$, G. Maret ${ }^{1}$, and P. Nielaba ${ }^{1}$

1 Physics Department, University of Konstanz, 78457 Konstanz, Germany

2 Institut für Physik, Johannes Gutenberg-Universität Mainz, Staudinger Weg 7, 55099 Mainz, Germany

${ }^{3}$ Graduate School Materials Science in Mainz, Staudinger Weg 9, 55128 Mainz, Germany

\begin{abstract}
A brief review focusing on low-dimensional colloidal model systems is given describing both simulation studies and complementary experiments, elucidating the interplay between phase behavior, geometric structures, and transport phenomena. These studies address the response of these very soft colloidal systems to perturbations such as uniform or uniaxial compression, laser fields, randomly quenched disorder, and shear deformation caused by moving boundaries.

Binary hard-disk mixtures are studied by Monte Carlo simulation, to investigate ordering on surfaces or in monolayers, modeling the effect of a substrate by an external potential. By weak external laser fields the miscibility of the mixture can be controlled, and the underlying mechanism (laser-induced demixing) is clarified. The stability of various space-filling structures is discussed only for the case where no laser fields are present.

Hard spheres interacting with repulsive screened Coulomb or dipolar interaction confined in 2D and 3D narrow constrictions are investigated by Brownian Dynamics simulation. With respect to the structural behavior, it is found that layers or planes throughout the microchannel are formed. The arrangement of the particles is disturbed by diffusion, and can also be modified by an external driving force causing a density gradient along the channel. Then the number of layers or planes gets reduced, adjusting to the density gradient, and this self-organized change of order also shows up in the particle velocities.

The experimental work that is reviewed here addresses dipolar colloidal particles confined by gravity on a solid substrate on which a set of pinning sites has been randomly distributed. The dynamics of the system is studied by tracking the trajectories of individual particles, and it is found that the mean square displacements of particles that are nearest neighbors of pinned particles are strongly affected by these
\end{abstract}


defects. The influence of the pinning sites on the order and microscopic mechanism of phase transitions in two dimensions is investigated.

\section{Introduction}

Soft matter with its structural and elastic properties offers an attractive route to the design of new materials. The importance of structured surfaces or monolayers lies in their promising, versatile technical applicability. Examples are antireflection surfaces, optical storage media or the usage in template-directed colloidal crystallization, the resulting three-dimensional colloidal crystals being, e.g., of interest due to their tailored photonic band gaps. The physics of surfaces and adsorbed monolayers has attracted a lot of interest in this context. In theoretical studies two dimensional systems are often used as a model, the interactions with substrates being conveniently modeled by external fields. The experimental counterpart, two-dimensional systems of colloidal suspensions, has been studied extensively in the last decades. They offer direct access to real space data via video microscopy, an excellent control over the colloidal interactions and easy tunability of the substrate potential in its shape and strength, as it is modeled, e.g., by interference patterns of laser beams. A close interplay between experiments, analytic theory, and computer simulations helped to shed light on such fundamental questions of statistical physics as the nature of melting in two dimensions with and without the influence of external fields. The interaction of monodisperse, two dimensional systems with one-dimensional, periodic light fields lead to the discovery of complex phase behavior as for example laser induced freezing and laser induced melting. Elastic properties of two-dimensional colloidal crystals have been analysed by us recently [1,2], as well as the effect of external fields on structures and phase transitions [3-7].

Particularly with respect to the interest in complex, two dimensional structures for the design of new materials and the rich phase behavior resulting from the interaction of monodisperse systems with simple external light fields, questions arise, as to what complex, periodic structures binary mixtures might assemble in, and what influence an interaction with simple external light fields has on the phase behavior of such mixtures. In [3] we focused in particular on the differences in the induced ordering and ordering mechanisms that result from the various possible interaction scenarios with the external potential in a mixture. Binary hard-disk mixtures are chosen as a model system for the clarification of these questions. Hard-disks are neither selective in the choice of the type of next-neighbor particles nor in the number of next-neighbor particles. Therefore the model system is ideal to show what ordering can result solely from geometric constraints due to the diameter ratio $\sigma_{B} / \sigma_{A}$ of the components of the mixture and due to the concentration of the mixture. These characteristics make them also a good model for the ground state of atomic systems with short range interactions.

In the present work, we focus on such low-dimensional colloidal model systems, formed from spherical particles, via simulation studies and also some complementing experiments, in order to elucidate the interplay between phase behavior, geometric structures, and transport properties. In particular, we try to investigate the (nonlinear!) response of these very soft colloidal systems to various perturbations: uniform and uniaxial pressure, laser fields, shear due to moving boundaries, and randomly quenched disorder.

The manuscript is organized as follows: In Sect. 2, a two-dimensional colloidal crystalline strip formed from point-like particles interacting with a repulsive potential (of power law form) confined between corrugated walls is considered. Structural changes induced by two types of deformation are studied: uniaxial compression can 
cause a transition from a structure that is commensurate with the boundaries to an incommensurate structure, characterized by "soliton staircases" along the walls; shear deformation, where the boundaries move in opposite directions, on the other hand, leads to grain boundary formation and plastic flow. In Sect. 3, a two-dimensional colloidal system formed from two types of disk-like particles is studied, where the crystal structure is not a simple triangular lattice like in Sect. 2, but more complex lattice symmetries occur. Uniform pressure as well as laser fields are used to control the structures and study possible transitions, such as laser-induced freezing, melting or demixing. Section 4 then considers transport of colloids in quasi-one-dimensional channels, which (unlike the system of Sect. 2) are only a few colloid diameters wide. Both channels in three-dimensional space (with a square cross section) and twodimensional channels are investigated. Transport is induced by a field acting along the axis of the channel. Also in this case, a periodic potential along the channel leads to very interesting effects, that are briefly reviewed here. Still another variation on our general theme is presented in Sect. 5, where experiments are described that elucidate the effect of quenched disorder on structure and dynamics of two-dimensional layers of spherical colloidal particles.

\section{Dynamics of colloidal particles in two-dimensional colloid crystals confined by corrugated walls: Effects of compression and shear}

\subsection{Introduction: Description of the chosen model}

Two-Dimensional Crystals are special, because (in the thermodynamic limit) they lack long-range positional order [8]. As a result, displacement-displacement correlation functions behave as $\left\langle[\mathbf{u}(\mathbf{r})-\mathbf{u}(0)]^{2}\right\rangle \propto \ln (r / a)$, for two particles a distance $r \gg a$ apart ( $a$ being the lattice spacing of the triangular lattice that results as the ground state of the crystal, assuming point particles interacting with a purely repulsive potential, such as $V(r)=\varepsilon(r / \sigma)^{-m}$ ( $\varepsilon$ being the strength and $\sigma$ the range of this potential); we shall take, in the following $m=12$ [9-12]).

When one confines such a crystal by (one dimensional) "walls", parallel to a close-packed lattice axis of the crystal, to generate a quasi-one-dimensional strip of width $D$, several cases need to be distinguished: (i) the confining boundaries can be smooth and structureless, or corrugated. (ii) In the latter case, the corrugation can be commensurate or incommensurate with the (ground state) crystal structure at the chosen particle density. (iii) The distance $D$ between the confining boundaries can be commensurate with the crystal structure, i.e. an integer number $n$ of rows fits in between the walls $(D=n a \sqrt{3} / 2)$ or they may have some mismatch, described by the "misfit" $\Delta\{D=(n-\Delta) a \sqrt{3} / 2$, for $\Delta>0$ the strip is compressed; note that we measure $D$ from the first row of mobile particles to the first row of the fixed particles in the upper wall, Fig. 1.

These different ways of confinement have characteristic consequences on both order and fluctuations of the particles in such crystalline strips [9-11]. In all cases, one finds that the positional order in the direction perpendicular to the walls is stabilized; mean-square fluctuations of particles in $y$-direction are reduced near the walls, [9$12]$, and a pronounced "layering" near the walls occurs already in the fluid phase at temperatures $T$ exceeding the melting temperature $T_{m}\left(k_{B} T / \varepsilon \approx 1.35\right.$ [13]). Note that the model studied in Refs. [9-13] does not depend on density $\rho$ and temperature $T$ separately, but only in the combination $X=\rho\left(\varepsilon / k_{B} T\right)^{1 / 6}$; in real colloidal suspensions the temperature is not a control variable, of course, but rather the density $\rho=N / V$ 


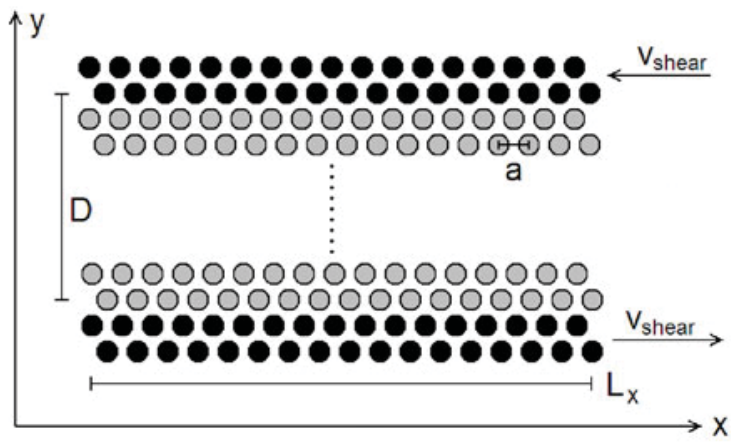

Fig. 1. Sketch of the geometry of a two-dimensional strip of mobile colloidal particles (gray circles) confined by two rows of particles frozen in a perfect triangular lattice structure (lattice spacing $a$; block circles). In $x$-direction the linear dimension is $L_{x}=n_{x} a$ and periodic boundary conditions are used. In the commensurate case, the linear dimension $D=n_{y}(a \sqrt{3} / 2)$, with $n_{y}=30$ rows of particles fitting into the strip. Arrows on top and bottom indicated shear motion induced by moving the walls with velocities $v_{\text {shear }}$ and $-v_{\text {shear }}$, respectively.

where $N$ is the total number of colloids and $V$ the available volume. The quoted value of $T_{m}$ refers to $\rho=1.05$ (choosing units such that $\sigma=1$ and $\varepsilon=1$ ). Note $\rho=2\left(\sqrt{3} / a^{2}\right)$ for the ideal triangular lattice. In the following we shall always discuss the behavior of the model for different temperatures (choosing $T$ in the range from $T=0.1$ to 1.0 , with $k_{B} \equiv 1$ ), but one should keep in mind that the variation of $T$ can be straightforwardly translated into a variation of $\rho$.

It turns out [9-12] that the variation of properties in the $x$-direction has a very interesting dependence on the different boundary conditions, mentioned above. Using corrugated walls that are commensurate with the crystal structure in $x$-direction, the crystalline order in the strip is stabilized. Then the displacement correlation function $\left\langle\left[u_{x}(i, j)-u_{x}(i+k, j)\right]^{2}\right\rangle$, where the index $i$ labels the particles in the $\mathrm{j}^{\prime}$ th row along the $x$-direction, saturates at finite values for $k \rightarrow \infty$, and also the mean square displacements relative to the ideal lattice positions $\left\langle\left[\Delta u_{x}(i, j)\right]^{2}\right\rangle$ all are finite. These quantities are all smallest near the corrugated walls and largest in the center of the strip (when the number of rows $n$ in the $y$-direction goes to infinity, we would cross over to the bulk two-dimensional crystal, where mean-square displacements are infinite, positional long-range order is destroyed). Note that these results also apply in case of nonzero misfit $\Delta$, as long as no transition in the number of rows has occurred [9-12].

If the confinement is produced by smooth linear boundaries $[9,10]$ rather than by the corrugated boundaries of Fig. 1, the system rather behaves quasi-one-dimensional: $\left\langle\left[u_{x}(i, j)-u_{x}(i+k, j]^{2}\right\rangle\right.$ is largest near the boundaries, and exhibits a crossover from the logarithmic variation $(\propto \ln k)$ to a linear variation $(\propto k)$, depending on the thickness $D$ of the strip [16]. Note that the linear variation with $k$ is well-known from harmonic chains [14]; the structure factor of such a one-dimensional model [14], which shows a smooth transition from fluid behavior at high temperature to a crystal at $T=0$, with a gradual increase of the correlation length for positional order in $x$-direction, provides also a reasonable description for the structure factor of the strip [9-11].

The most interesting behavior occurs for corrugated walls and large misfits [12,15-18]. Then the system undergoes a transition from $n$ to $n-1$ rows (and upon further compression to $n-2$ rows [18] etc.), and the particles of the row that 


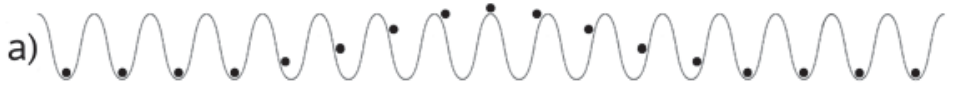

b)
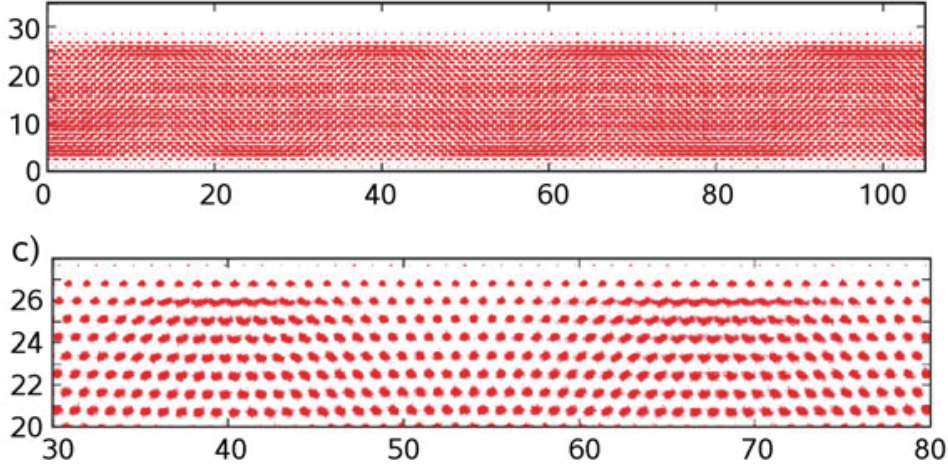

Fig. 2. (a) Illustration of a soliton configuration: putting $n+1$ particles in a periodic potential where $n$ minima creates a misfit, where over a range of several lattice spacings particles are displaced from the potential minima. (b) Superimposed snapshot pictures of 750 configurations of the particle positions where for a system of $n_{y}=30$ rows and a large misfit $(\Delta=2.6)$ a transition to $n_{y}-1=29$ rows has occurred $\left(n_{y}=108\right.$ and $T=1.0$ were chosen). The four solitons at each wall are visible due to the larger lateral displacements of the particles, leading to a darker region in the snapshot. Panel (c) shows a close up of the structure near the upper wall. Numbers shown along the axes indicate the Cartesian coordinates of the particles. Panels (b) and (c) have been adapted from Chui et al. [15]. [=Fig. 3 of PRE 85, 056702 (2012)].

disappears at such a transition need to be re-distributed in the remaining rows, which then have a somewhat smaller lattice spacing also in $x$-direction; hence the commensurability with the corrugated walls is destroyed. This leads to a formation of a "soliton staircase" pattern [19] (Fig. 2). Due to the accompanying standing strain density wave there is a long-range elastic distortion of the whole crystalline strip, and hence the minimum free energy state is obtained for this regular arrangement of four solitons at each wall (Fig. 2b). Note that the $n=108$ extra particles of the row that is removed in the $n_{y} \rightarrow n_{y}-1$ transition are hosted only in the $n_{y}-3=27$ inner rows of the strip (no extra particles go into the rows directly adjacent to the rigid boundary rows). Therefore, the number of solitons at each wall simply is $n_{x} /\left(n_{y}-3\right)(=4$, in the example shown in Fig. 2b). In the center of the strip, it is energetically favorable to create a uniform compression of the lattice spacing in $x$-direction rather than this nonuniform soliton stair case pattern, which only results at the walls due to the periodic potential created by the boundary particles. For this incommensurate structure this periodic potential no longer has a stabilizing effect, and thus mean square displacements $\left\langle\left[\Delta u_{x}(i, j)\right]^{2}\right\rangle$ do not exist (i.e., are infinite), similar to the case of confinement by planar walls.

\subsection{Local motions versus diffusion}

For simplicity, the dynamics of the colloidal particles is simulated using the Newtonian equation of motion ( $m \equiv 1$ is the mass of a colloidal particle)

$$
m d \mathbf{v}_{i} / d t=\mathbf{F}_{\text {pot }}+\mathbf{F}_{\text {fric }}+\mathbf{F}_{\text {rand }}, \quad \mathbf{F}_{\text {pot }}=-\partial V_{\text {tot }}\left(\mathbf{r}_{i}\right) / \partial \mathbf{r}_{i}
$$



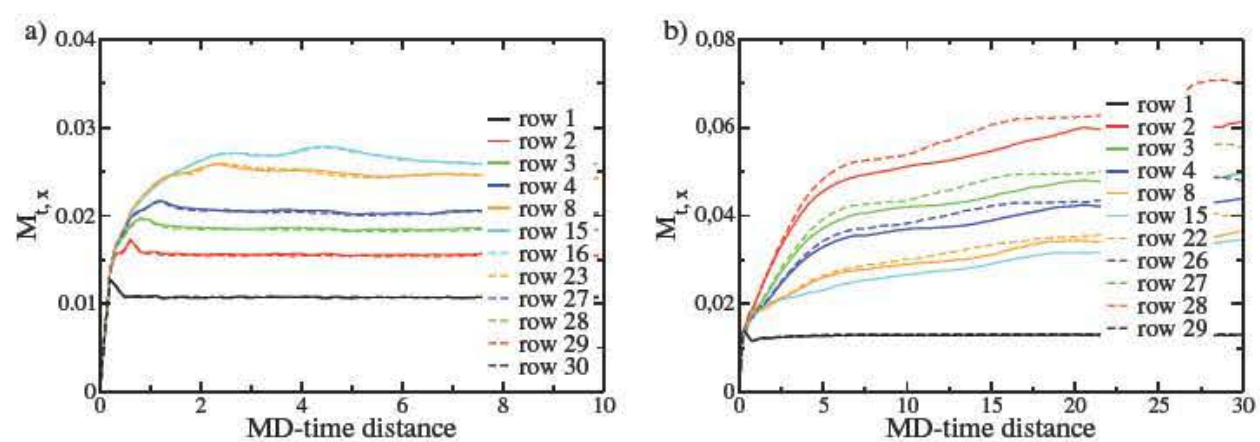

Fig. 3. Plot of $M_{j x}(\Delta t)$ versus the MD time distance $\Delta t$, for a compressed but commensurate system with $n_{y}=30$ rows $\left(\Delta=1.5\right.$., case (a)) and an incommensurate system $\left(n_{y}=29\right.$ rows, $\Delta=2.2$., case (b)). $T=1.0$ in both cases. Curves label the particles in the different rows. From Wilms et al. [24] [=Fig. 6(a) and Fig. 8 of PRE86, 051404 (2012)].

where $V_{\text {tot }}\left(\mathbf{r}_{i}\right)$ is the total potential acting on the i'th colloid due to its interaction with other particles. The friction force is linear in the velocity $\mathbf{v}_{i}, F_{\text {fric }}=-\gamma \mathbf{v}_{i}$, and the friction coefficient $\gamma$ satisfies the usual fluctuation-dissipation relation with the random force, in $d=2$ dimensions

$$
\left\langle\mathbf{F}_{\text {rand }}(\mathbf{r}, t) \cdot \mathbf{F}_{\text {rand }}\left(\mathbf{r}^{\prime}, t^{\prime}\right)\right\rangle=4 \gamma T \delta\left(\mathbf{r}^{\prime}-\mathbf{r}\right) \delta\left(t-t^{\prime}\right) .
$$

Note that Eqs. (1), (2) neglect hydrodynamic interactions, but this neglect should be legitimate at the high densities of interest [20]; even for somewhat less dense colloidal suspensions in the fluid phase close to the transition to the glassy state hydrodynamic interactions have already only negligible effects, as the perfect agreement of experiments with the mode coupling theory demonstrates (see $[21,22]$ for a review). We typically use $\gamma=0.5$, and for this strong damping the inertial term in Eq. (1), which only is included for computational convenience, has an effect only for very short times, $t \leq 1 \tau_{M D}=\left(\varepsilon / m \sigma^{2}\right)^{1 / 2}=1$. We apply the velocity Verlet algorithm using the HOOMD-blue package [23].

Local motions now are conveniently monitored by studying mean square displacements as function of time, $M_{j, x}(\Delta t) \equiv\left\langle\left[x_{j}(t)-x_{j}(t+\Delta t]^{2}\right\rangle\right.$, where the average is over $t$ as well as over all particles in row $j$. Figure 3 compares the behavior of $M_{j x}(\Delta t)$ for an incommensurate case $(\Delta=2.2)$ with a commensurate one $(\Delta=1.5)$. One notes that in the commensurate case $M_{j x}(\Delta t)$ quickly settles down at a constant, the static mean square displacement $\left\langle\left[\Delta u_{x}(i, j)\right]^{2}\right\rangle$. At short times, there is an overshoot (interpreted as a damped oscillation motion of a particle in a cage formed by its neighbors). In contrast, in the incommensurate case a plateau occurs only for the two rows adjacent to the walls (because they stay commensurate). In the other rows the $M_{j, x}(\Delta t)$ quickly (on a time scale of a few $\tau_{M D}$ ) increases to a shoulder, and then crosses over to a slow linear increase with time (indicative of the fact that $\left\langle\left[\Delta u_{x}(i, j)\right]^{2}\right\rangle$ does not exist, since there is a lack of positional long range order). Note that in the incommensurate case the rows where the solitons occur, adjacent to the walls, exhibit the largest increase, while in the commensurate case the largest $M_{j x}(\Delta t)$ did occur in the center of the strip [12].

Apart from the local motions displayed in Fig. 3, also diffusion of particles occurs, even in the commensurate case where $\left\langle\left[\Delta u_{x}(i, j)\right]^{2}\right\rangle$ is finite [12]. Two mechanisms of diffusion have been identified: one mechanism is the standard nucleation of vacancyinterstitial pairs [24], the other mechanism is a collective random rotation of groups of particles around a center. E.g., six nearest neighbors of a particle in the center of 

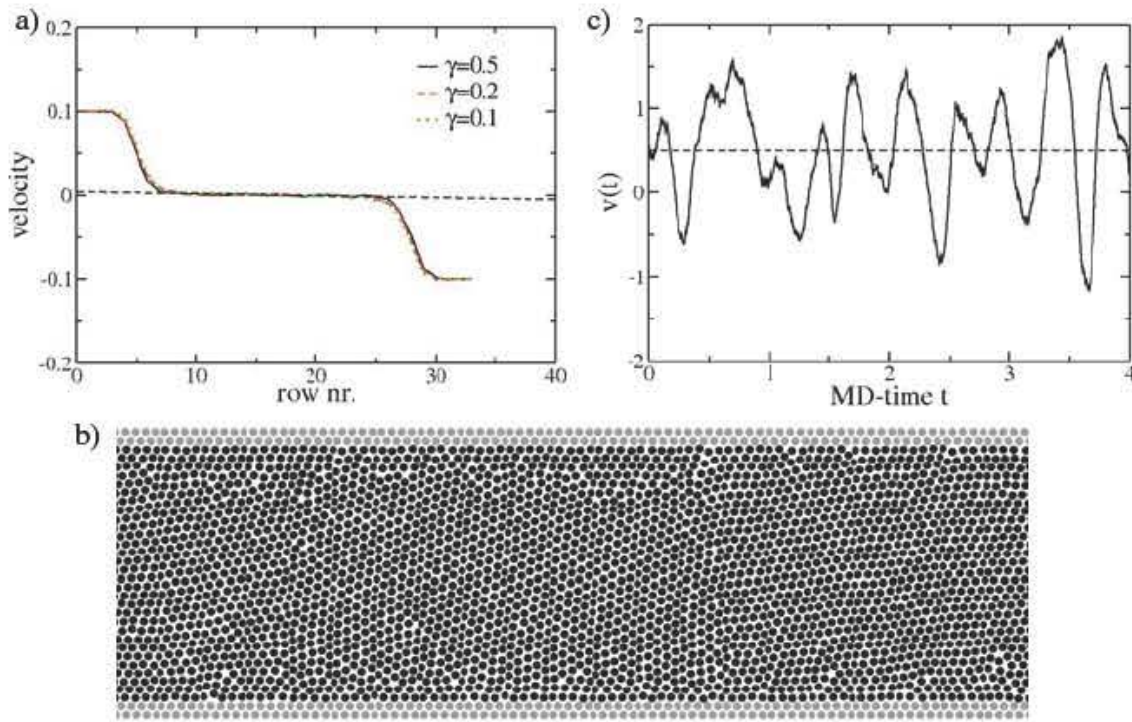

Fig. 4. (a) Profile of the average velocity $v(y)$ across the film, with $y=j a \sqrt{3} / 2, j=$ $0,1 \ldots, 33\left(j=0,1\right.$ and 32,33 represent the rigid walls), for $T=0.3 ., \Delta=0, v_{\text {shear }}=0.3$. Three choices of friction coefficient are included [=Fig. 3 (a) of [26]] (b) Snapshot of a section of the system used in (a) [=Fig. 4, upper part of [26]]. (c) Time-dependent velocity of a particle in the row $j=2$ adjacent to the moving wall. [=Fig. 5(b) of [26]].

the hexagon formed by these neighbors "rotate" almost coherently (either clockwise or anticlockwise: both choices occur, at random) by about $60^{\circ}$. Also similar collective motions of groups of neighboring particles forming a triangle or quadrumer occur. But while such collective motions, which do not require the nucleation of vacancyinterstitial pairs, displace only a few (3-6) particles by a lattice unit, the diffusion of a vacancy through the lattice displaces of the order of 100 particles, before typically it annihilates by meeting the corresponding interstitial particle again (at the temperature of interest in the finite system of typically 64800 mobile particles only one vacancy-interstitial pair occurs at at time). Studying the number of such events as a function of temperature, we have estimated the activation energy to create vacancyinterstitial pairs, $E_{a} \approx 17.7$, while for ring rotation events $E_{a} \approx 14$. While the latter thus have a somewhat smaller activation energy, they displace much less particles; thus at most temperatures of interest the standard vacancy-interstitial mechanism will dominate the self-diffusion of colloidal particles [12].

\subsection{Shearing the crystal: Plastic flow and stick-slip motion}

Particularly interesting phenomena occur when one moves the rigid boundaries with opposite velocities $\pm v_{\text {shear }}$ against each other (Fig. 1), depending on the magnitude of $v_{\text {shear. }}$ Typically for small enough magnitude of this shear in the commensurate case a few layers commensurate with the moving walls, move together with them at the same speed $[25,26]$. Then irregular grain boundaries occur, where the configuration of particles is fluid-like, and steadily rearranging during the motion (Fig. 4). In the central region of the strip large crystalline domains occurs, but the orientation of their crystal axes are systematically mis-oriented, in comparison to the commensurate system at rest. These domains are not completely static - there is also an average 
motion in the interior of the strip, described by $v(j)=0.1 v_{\text {shear }}(1-(j-1) / 31)$, see the straight line in Fig. 4a, for $8 \leq j \leq 26$. In the regime where $v(j)$ rapidly decreases, there is a lot of friction occurring, "heat" (i.e., kinetic energy of the colloidal particles) is produced, the system is far from equilibrium in these grain boundary regions. Analyzing the frequency spectrum of the local motions, that show irregular velocity oscillations (Fig. 4c), one finds a strong broadening in the rows corresponding to these rearranging grain boundaries. If one moves the corrugated wall very fast, however, the mobile particles cannot follow any longer the corrugated wall, the crystalline strip stays almost intact. For the incommensurate case, however, an important difference is that for small $v_{\text {shear }}$ we simply have $v(j)=0$ in the center of the strip, the rows where at rest the solitons occur act as lubricant (although the solitons get destroyed when the corrugated walls move).

Many of these observations have close similarities to the behavior known for friction of surfaces of atoms sliding against each other [27]. The distinctive feature of colloidal systems, of course, is that it is experimentally (and not only in simulations, Fig. 4c) possible [28] to follow the motion of single colloidal particles during friction and plastic flow.

\section{Binary two-dimensional model colloids in external fields}

In Ref. [3] we analyzed the properties of binary, two-dimensional hard-disk mixtures with $N=N_{A}+N_{B}$ particles by Monte Carlo simulations. The diameter of the larger component of the mixture was set to $\sigma_{A}=1$, and all lengths are measured in units of $\sigma_{A}$. The packing fraction of the mixture is defined as $\eta=\rho^{*} \pi\left(N_{A} \sigma_{A}^{2}+N_{B} \sigma_{B}^{2}\right) / 4 N$, where $\rho^{*}=\rho \sigma_{A}^{2}$ is the dimensionless number density. The concentration of large particles in the mixture is given by $x_{A}=N_{A} / N$. Periodic boundary conditions are used in all simulations, and besides the standard Metropolis algorithm a cluster move by Lue [29] has been employed.

\subsection{High pressure phases without external fields}

Simulations [3] in the NpT ensemble were used for testing the thermodynamic stability of given lattice structures. Here the simulation volume can fluctuate although shearing the simulation box was not attempted. In these studies suggested space-filling lattice structures for various binary mixtures [30] were set up with $N \approx 1000$. Simulations were run starting from a high, hydrostatic external pressure $p^{*}=p \sigma_{A}^{2} / k_{B} T$ and lowering it in successive runs.

An overview of the simulated binary mixtures is given in Fig. 5. For illustration sketches of the studied lattice structures are shown. Also given are the packing fraction $\eta$ and the pressure $p^{*}$, above which all of the simulated mixtures were found to be stable for the given lattice structures. Figure 5 shows that the more a given lattice structure deviates from the triangular, monodisperse lattice the higher the hydrostatic pressure needed to stabilize it. The simulations show that the complex, space-filling lattice structures for binary hard-disk mixtures are high pressure phases. This makes it difficult to observe such structures in experimental set ups, as by increasing the pressure within the surface the probability that the monolayer will escape into the third dimension by buckling will also increase. These findings show clearly the importance of the search for alternative ways to stabilize or induce the formation of ordered structures in two dimensional, binary mixtures. One promising approach is the use of external fields. 


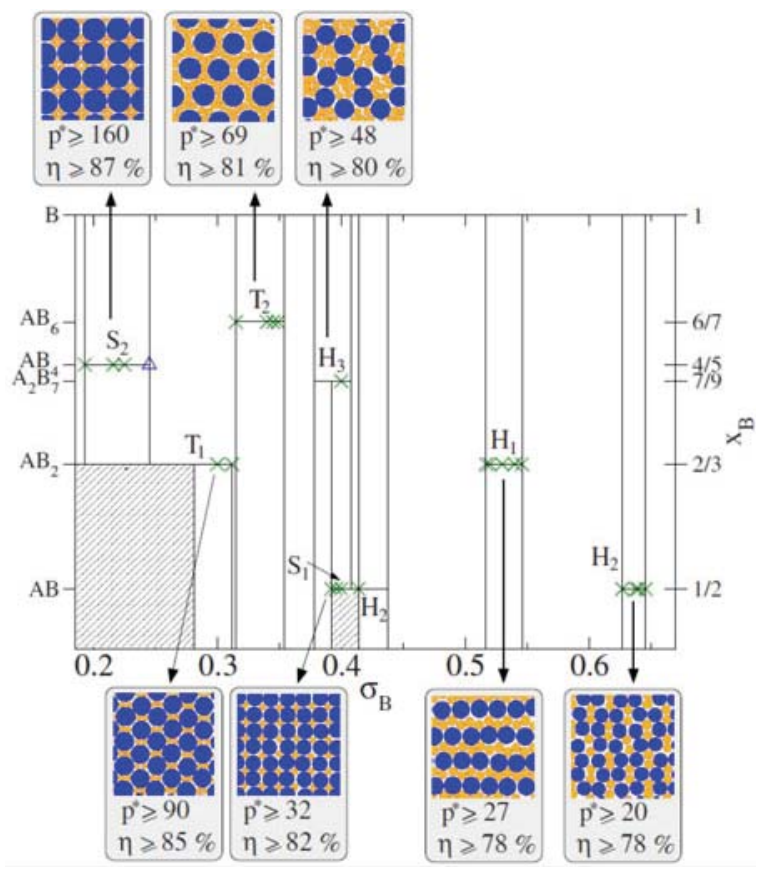

Fig. 5. Overview of analyzed lattice structures for binary hard-disk mixtures. Structures for which a pressure regime, in which the structures stay stable, could be identified are marked by crosses (green). For the mixture marked by a triangle (blue) the lattice structure was not stable within the simulated pressure regime of $p^{*} \leq 200$. Also given are the packing fraction $\eta$ and the pressure $p^{*}$ above which all of the simulated mixtures for the given lattice structure were found to be stable. After Franzrahe et al. [3].

\subsection{Effects of external fields}

Monodisperse 2D colloidal systems in interaction with a substrate potential have been studied extensively in experiments [31,32], computer simulations [5, 6,33,34] and theory [35-37] over the last decades. Reentrant phase transition scenarios like Laser Induced Freezing (LIF) and Laser Induced Melting (LIM) have been observed. In a recent study [3] we addressed the question, how the addition of another length scale into such a system influences the intricate competition between adsorbate-adsorbate and adsorbate-substrate interaction by studying a binary $50 \%$ mixture under the influence of a 1D spatially periodic substrate potential. The colloids are modeled by hard disks with a diameter ratio $\sigma_{B} / \sigma_{A}=0.414$. Following the approaches in the monodisperse studies, we use the following external potential: $V(\mathbf{r})=V_{0} \sin (\mathbf{K} \cdot \mathbf{r})$ with $\mathbf{K}=(4 \pi / a, 0)$ and $a$ the lattice parameter of the $S_{1}(A B)$ lattice. Thus the wavelength $\lambda=2 \pi /|\mathbf{K}|$ of the external potential is commensurate to the periodicity of the $S_{1}(A B)$ lattice.

The influence of a modulated external field was analyzed [3] by Monte Carlo simulations in the NVT ensemble. In these simulations additional nonlocal moves are attempted with particle displacements, which are integer multiples of the potential wavelength. The simulation box is set up to be slightly rectangular with $L_{x} / L_{y} \approx$ 1.178 and $N=1848$ for these simulations. This choice allows not only a box-spanning square lattice to form, but also a box-spanning monodisperse triangular lattice of the larger component can develop. After equilibrating the system for $10^{7}$ Monte Carlo steps (MCS) the simulations were run for another $10^{7} \mathrm{MCS}$ and data were taken. 


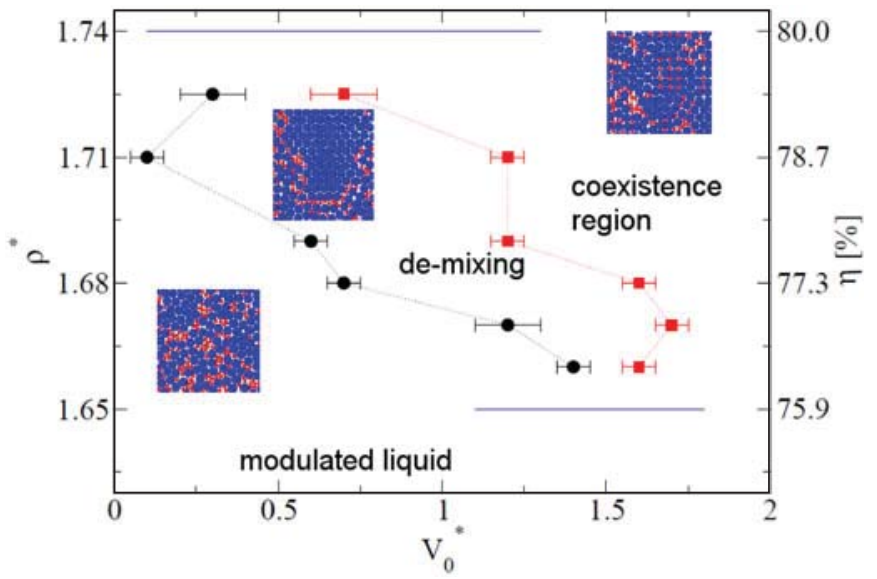

Fig. 6. The Laser Induced Demixing regime of the phase diagram for an equimolar binary mixture with diameter ratio $\sigma_{B} / \sigma_{A}=0.414$ exposed to an external periodic potential, commensurate to the $S_{1}(A B)$ square lattice for the case that only the smaller component of the mixture interacts with the external potential. After Wilms et al. [38].

For the interaction of the mixture with the external field three cases can be distinguished: (I) only the smaller component interacts with the external field [4], (II) both components interact with the external field, (III) only the larger component interacts with the external field.

For low amplitudes $V_{0}^{*}=V_{0} / k_{B} T$ we found a novel phenomenon: in contrast to the monodisperse LIF scenario a Laser Induced Demixing [4] sets in for all three cases. Laser Induced Demixing results in the coexistence of a small component enriched fluid with a droplet of a mono- disperse crystalline structure formed by the larger component. The ordering mechanisms at work and the resulting monodisperse lattice structures differ for the three cases [3]. The ordering mechanisms stem from the attempt of the system to minimise its energy via an alignment of the components interacting with the external potential with the potential minima and the constraint of a fixed overall number density. While the coupling of only the smaller component to the external potential (case (I)) results in the coexistence of a monodisperse triangular lattice, the cases (II) and (III), where the larger component interacts directly with the external potential, show a condensation of a monodisperse rhombic lattice.

In Fig. 6 we present $[3,4,38]$ the phase diagram and configuration snapshots for the Laser Induced Demixing regime for the case (I). Above and below the horizontal blue line no Laser Induced Demixing was observed. The lines in the phase diagram show the phase boundaries between the modulated liquid and the demixing regime as well as the boundary to a regime where square lattice structures and the demixed monodisperse structures compete.

\section{Transport in colloidal model systems}

Non-equilibrium stationary states have received much attention recently in different areas of science ranging from the analysis of pedestrian dynamics [39], to biologically relevant transport of ions in membranes [41], to lab-on-chip devices and microfluidics [40]. The behavior of colloidal systems [42] has been studied both in two-dimensional (2D) [43-45] and in three-dimensional (3D) systems [46-53]. Experiments for non-equilibrium structure formation have been done with oppositely 
charged colloids driven in opposite directions by an electric field [54] or in binary complex plasmas under microgravity conditions [55]. Such driven, diffusive systems serve as model systems for the theoretical studies of non-equilibrium behavior [56].

Here we present results for the structure and dynamics of colloidal systems confined to two- and three-dimensional channels in equilibrium and under the action of external constant forces. Details of the model and methods have been presented elsewhere [57].

The time evolution of the system is obtained by Brownian Dynamics simulation, based on the overdamped Langevin equation. Hydrodynamic interactions as well as short time momentum relaxation of the particles are neglected. The for this situation appropriate stochastic position Langevin equation with friction constant $\xi$,

$$
\xi \frac{d \mathbf{r}_{i}(t)}{d t}=-\nabla_{\mathbf{r}_{i}} \sum_{i \neq j} \mathcal{V}_{i j}\left(r_{i j}\right)+\mathbf{F}_{i}^{(e x t)}+\tilde{\mathbf{F}}_{i}(t)
$$

is time-integrated with a finite time step $\Delta t$ and the technique by Ermak [58]. The right-hand side includes the particle interaction as a sum over all forces acting on each particle, the constant driving force $\mathbf{F}_{i}^{(e x t)}$ and the random forces $\tilde{\mathbf{F}}_{i}$, describing the random collisions of the solvent molecules with the $i$-th colloidal particle and modeled as a white noise term, representing a heat bath with temperature $T$. The 2D channels have a length of $L_{x}=800 \sigma$ and a width of $L_{y}=10 \sigma$ containing 3200 particles corresponding to a density of $\rho=0.4 \sigma^{-2}$. The 3D channels typically have a quadratic square section of $L_{y}=L_{z}=5 \sigma$ and a length of $L_{x}=1000 \sigma$ containing about 10000 particles with a fixed particle density of $\rho=0.4 \sigma^{-3}$. The particles are confined within the channels by ideal hard walls. The equilibrium configuration in a closed channel is calculated starting from a random configuration and applying hard wall boundary conditions in all directions. The systems are typically equilibrated over $2 \times 10^{5}$ time steps. The time step $\Delta t=7.5 \times 10^{-5} t_{B}$ is used where $t_{B}=\xi \sigma^{2} / k_{B} T$ is the time a particle needs in equilibrium to diffuse its own diameter $\sigma$. The friction constant $\xi$ is chosen to be $\xi=3 \pi \eta \sigma=4,288 * 10^{-8} \mathrm{Ns} / \mathrm{m}$ where $\eta=0.001 \mathrm{~Pa}^{*} \mathrm{~s}$ is the shear viscosity of water. The simulations are done at a constant temperature $T=295 \mathrm{~K}$. To study the influence of an external force, a constant driving force of $F^{(\mathrm{ext})}=2.603 * 10^{-15} \mathrm{~N}$ is applied in longitudinal direction. The channel end is then realized by an open boundary condition. To keep the number density in the channel fixed a new particle is inserted within the first $10 \%$ of the channel at a random position avoiding overlaps every time a particle leaves the open end of the channel acting as a particle reservoir. This procedure seems to be appropriate to permit comparison to experimental studies $[61,62]$, in which the channel is coupled to two reservoirs.

Due to a confinement, a classical $2 \mathrm{D}$ system forms a layered structure in equilibrium $[9,59,61,62]$, and the change in the number of layers due to the geometry of the confinement has been predicted using Langevin Dynamic simulations [60]. The effect of the special boundary conditions in $x$-direction has been analyzed in great detail for two-dimensional systems in Ref. [62]. A constant driving force in $x$-direction and periodic boundary conditions would result in a layer formation for certain channel widths, similar to the equilibrium situation. The insertion of particles, which leave the channel end, at a randomly chosen place in the $10 \%$ region of the beginning of the channel results in a density gradient across the channel. At places along the channel, where the density has decreased sufficiently to energetically stabilize configurations with one layer less, the number of layers changes by -1 . This scenario is essentially the same for three-dimensional systems, except that the number of planes parallel to the $3 \mathrm{D}$-channel walls gets reduced instead of the number of layers. In case the external forces are not acting on the particles in the "reservoir regions" of the channel 


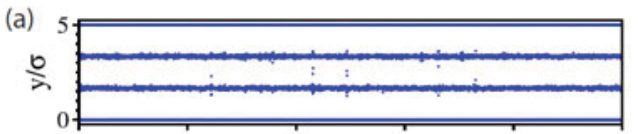

(b)

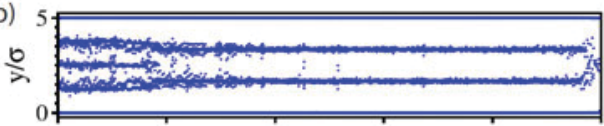

(c)
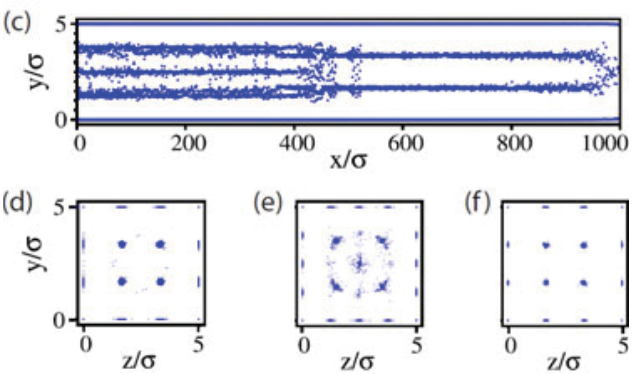

Fig. 7. Simulation snapshots of the configuration in a $3 \mathrm{D}$ microchannel (a) in equilibrium without an external force, (b) after $10^{5}$ time steps with an external driving force and (c) in the stationary non-equilibrium after $10^{6}$ simulation steps with a constant position of the plane reduction zone within the channel. For clarity all particle positions are projected on the $x y$-plane and the distances in $y$-direction are stretched by the factor 16. After Schwierz et al. [57].

(the first and last $10 \%$ of the channel), interestingly a density increase results in the flow direction [62].

\subsection{Transport behavior of colloids in 3D microchannels}

Now we address the transport behavior of colloids confined in a 3D microchannel. The colloids are driven by the application of an external driving force. First, we focus on the effect of a reduction of the number of planes i.e. the dynamical rearrangement of the colloids during their flow along the channel. This phenomenon is analogous to the layer reduction observed in the $2 \mathrm{D}$ microchannels [61,62]. Figure 7 a shows the equilibrium configuration in a $3 \mathrm{D}$ channel with a quadratic square section of width $w=5 \sigma$ and a length of $1000 \sigma$. In equilibrium a loose structure with four planes forms which is stretched perpendicular to the confinement and in which the particles can move away from the wall easily. In the stationary non-equilibrium the average drift velocity is $1.43 * 10^{-7} \mathrm{~m} / \mathrm{s}$ which is significantly larger than the drift velocity of non-interacting particles indicating non-plug-flow. Figure 7 shows the time evolution of the system: In equilibrium a plane structure forms with four planes parallel to the confining walls in $y$ - and $z$-direction. The external force leads after $10^{5}$ time steps to an additional fifth plane in the reservoir. The area with five planes moves with increasing time in the flow direction. Close to the end of the channel the number of planes reduces from four to three planes. After $10^{6}$ time steps the system reaches a stationary non-equilibrium. After that time the position, at which a reduction of the number of planes occurs, does not move in the flow direction any more. The transition point oscillates back and forward within a small area. The particles move in planes and layers adapting to the external force. At the transition point the particles have to switch from the mid-plane to one of the adjacent planes closer to the channel wall. Often, particles leave the mid-plane, proceed in the flow direction before changing 

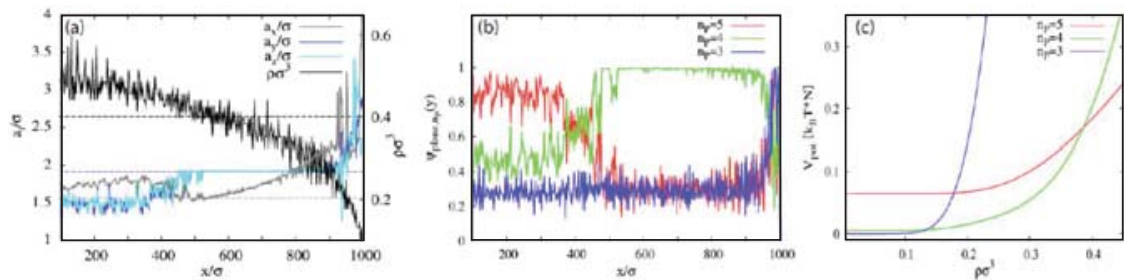

Fig. 8. (a) Local density distribution within the channel and lattice constants $a_{x}, a_{y}$ and $a_{z}$ in the stationary non-equilibrium after $10^{6}$ time steps. The dashed lines show the equilibrium values of the local density and the lattice constants. (b) Plane order parameter of the configuration shown in Fig. 7c. (c) Potential energy for a fcc-lattice in a channel of width $5 \sigma$ in dependence of the local density for the number of planes in the stationary non-equilibrium. After Schwierz et al. [57].

on a plane further outside. The local particle densities and the lattice constants are shown in Fig. 8a and the number of planes is shown in terms of the plane order parameter [57] in Fig. 8b. In the stationary non-equilibrium a density gradient forms along the complete length of the channel causing the change of the number of planes and therefore leading to a change of the lattice constants. At the left end of the channel the lattice constant $a_{x}$ is larger then $a_{y}$ and $a_{z}$ and the lattice is compressed perpendicular to the confinement. In the transition area $a_{y}$ and $a_{z}$ increase in two steps in contrast to the $2 \mathrm{D}$ systems where the lattice constant $a_{y}$ shows a single jump. At the end of the transition area $a_{x}$ is smaller than $a_{y}$ and $a_{z}$. While the latter two remain constant until the next transition point, $a_{x}$ increases monotonically. This behavior suggests, that stretching of the lattice in the flow direction leads to an instability causing the reduction of the number of planes. The change in the number of planes is clearly visible from the plane order parameter shown in Fig. 8b. The order parameter does not have a sharp transition point but rather a transition area with an extension of about $100 \sigma$ in which the value changes step like. The reason is that the order parameter of the wall particles drops to zero about $100 \sigma$ later than the order parameter of the planes in the middle of the channel. Thus, the planes closest to the wall are more stable against a deformation of the lattice. Again, the reduction of the number of planes can be explained by calculating the potential energy per particle in dependence of the local density. Starting from an ideal fcc-crystal with a constant number of planes, i.e. constant distances between the particles in $y$ - and $z$-direction, the distances in $x$-direction are increased or decreased and the potential energy is calculated. The dependence of the potential energy on the local density for a constant number of planes is shown in Fig. 8c. Above a value of the local density of $\rho \sigma^{3}=0.38$ it is energetically favorable for the system to form five planes, for values $0.38>\rho \sigma^{3}>0.13$ four planes and below $\rho \sigma^{3}=0.13$ three planes. These values are slightly smaller than the values at which a reduction occurs in the simulations. This can be explained by the fact that the density in the middle of the channel is smaller than close to the walls.

\subsection{Diffusion of model colloids in 2D microchannels}

The effects of confined geometry and of pinning sites on the diffusion of particles have been studied here and in the next section (experiment). In both situations, a subdiffusive behavior has been found for certain time regions. In a recent work [67], in collaboration with the experimental group of project C1 of the SFB TR6, we have studied the effect of the layering in two-dimensional colloidal system on the diffusion 
behavior. At a constant dimensionless interaction strength and constant density, layered systems and more disordered systems alternate with increasing channel width $L_{Y}$, as described in the previous section. This type of layering order can also be characterized by a layer order parameter $\Psi_{\text {layer, } n_{l}}[57,61,62]$, which is near 1 if the particles are structured in $n_{l}$ equidistant layers and otherwise much smaller. This layering has an important influence on the diffusion, which can be characterized by the mean square displacement (MSD), $\left\langle\Delta \mathbf{r}^{2}(t)\right\rangle=\frac{1}{N} \sum_{i=1}^{N}\left|\mathbf{r}_{i}(t)-\mathbf{r}_{i}(0)\right|^{2}$, where the sum runs over all particles $N$. For the diffusion in $x$-direction only the $x$ coordinates are considered in $\left\langle(\Delta x(t))^{2}\right\rangle$.

Starting from the well known case of single file diffusion [66] at channel width $L_{Y}=1$ with a long time evolution of the MSD $\left\langle(\Delta x(t))^{2}\right\rangle \propto t^{0.5}$, we studied [67] the dependency of the MSD on the width of the channels. The MSD for wider channels has a non-monotonic dependency on the width $L_{Y}$. This non-monotonic behavior is induced by the layering effect as described above. The diffusion has a maximum for disordered channels and a minimum for channels with layering.

In the long time limit, the standard diffusion exponent 1 is found. However, for channel widths stabilizing the layer structures, on intermediate time scales the MSD has a time dependency which can be characterized by an effective exponent of 0.5 over a long time span. Due to the length of these time spans this effective exponent is of great experimental relevance.

\subsection{Transport across a periodic potential}

In a recent work [64] we study the influence of a periodic potential in x-direction and external driving force $F$

$$
V_{\text {ext }}=V_{\text {period }} \sin \left(\frac{2 \pi}{\lambda} x\right)+F x
$$

with strength of the periodic potential $V_{\text {period }}=10 k_{B} T$ and period $\lambda$ equal to the layer spacing $R$. We consider a commensurate system $L_{Y}=5.2$ with four rows. Applying the periodic potential, we now have two competitive order principles, the layering parallel to the wall and the preference of the particle to be located in the potential minimum orthogonal to the walls. In the presence of a periodic potential the layering is suppressed. The ordering changes from a layering to a situation, where in each minimum either two, three or four particles are located Fig. 9b, c. The border particles are still well aligned at the wall, and also the density at the border is slightly increased. The two mid rows do not exist anymore.

If an external force acts in channel direction the particles start to hop over the barrier into the next minimum, which can be seen from the velocity of a single particle (see Fig. 9e). For $F=20$ there are only very rare hopping events, where for $F=30$ the particles already hop quite regularly. For $F=40$ and higher forces the motion can be described by a stick-slip motion instead of a succession of hopping events. This transition in the mobility is attended by change in the order. The particles are again ordered in layers, which can be explained with the fact, that the particles only feel an averaged potential and so the effect of the boundaries again becomes dominant when the particles move.

In addition to the transport properties in one-directional force fields, in a recent study [65] the effect of counterflow, with forces driving half of the particles in opposite direction to the other half, on the non equilibrium structure formation has been studied in three dimensional channels. Interesting segregation phenomena have been found with segregation of the system into regions with the same particle velocity. In order to analyze the effect on different length scales, a segregation order parameter 

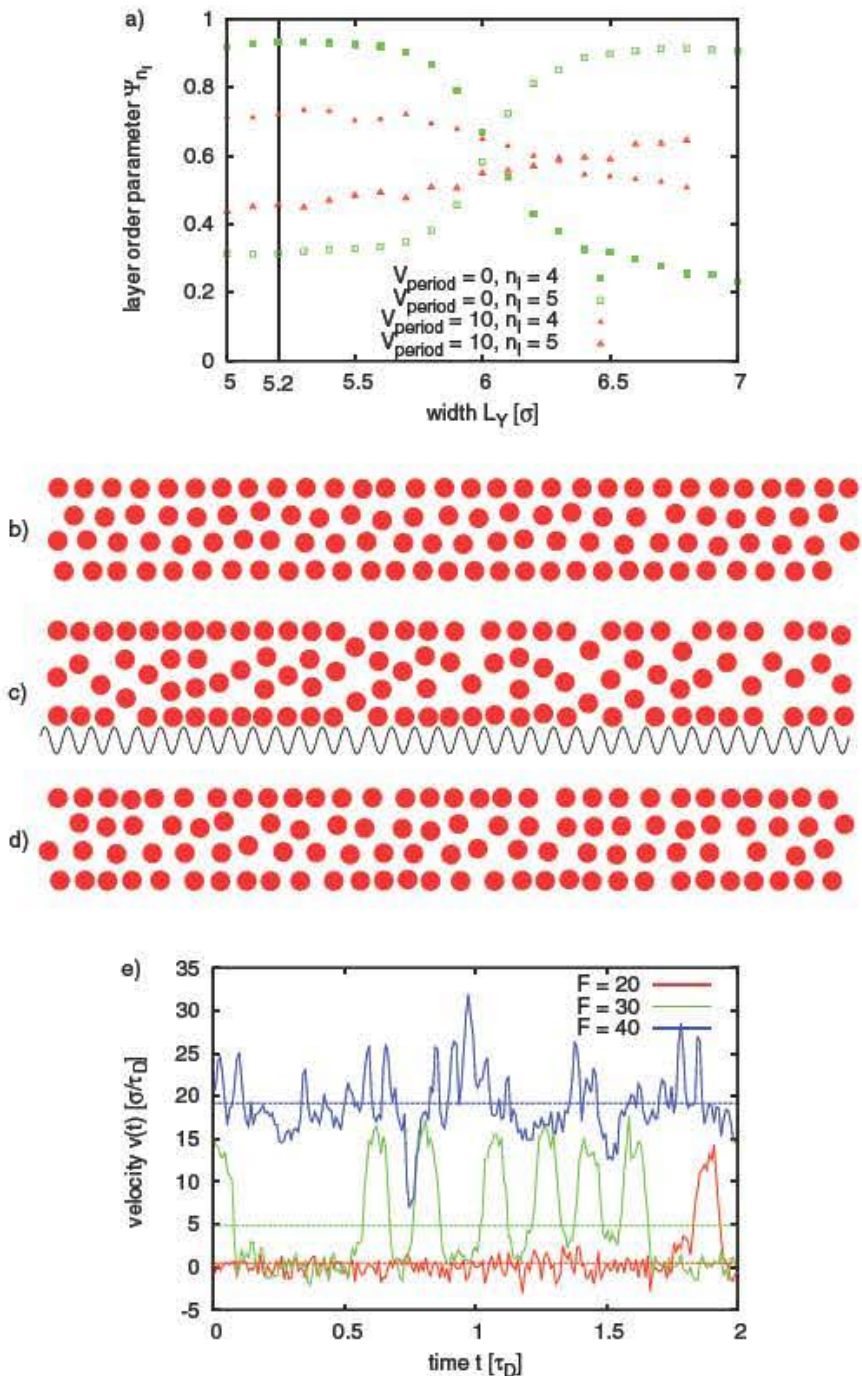

Fig. 9. (a) Layer order parameter as a function of channel width $L_{Y}$ with and without a periodic potential in x-direction. Snapshots of channels of width $L_{Y}=5.2$ (b) with no periodic potential, (c) with periodic potential and (d) with periodic potential and external force in x-direction of $F=40$. (e) Time-dependent velocity of a particle in a channel with with periodic potential and external force $F=20,30,40$.

has been computed in local analysis regions of various lengths d (see Fig. 10) in the channel direction. By a finite size scaling analysis it was found, that for constant external driving forces the segregation effect is higher on smaller length scales, see Fig. 10.

\section{Effect of quenched disorder on local dynamics and structure}

\subsection{Introduction}

The effect of quenched disorder on the melting of two-dimensional solids has been a fruitful debate for the last three decades. On the one hand, analytic approaches 


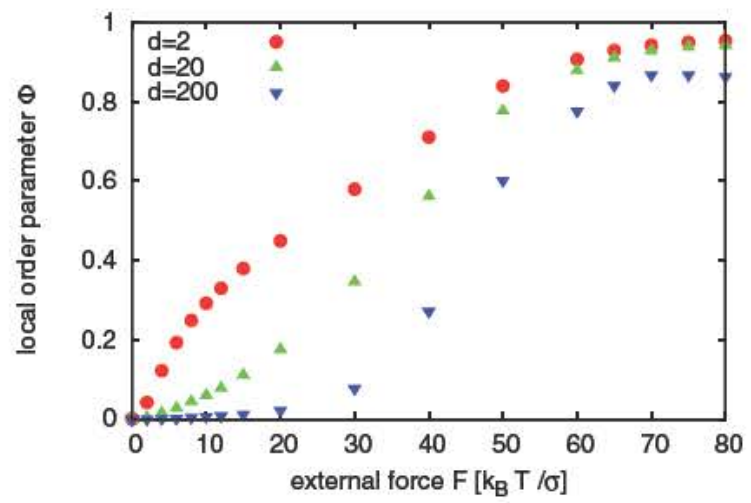

Fig. 10. Local order parameter for segregation as a function of external force in three dimensional channel of length $L_{x}=1000$ with counterflow. $d$ is the length scale of the local analysis region.

predict a KTHNY-like melting scenario for long wavelength disorder, and a sharp crossover between a liquid and a glassy regime if disorder varies on short range [68-70]. On the other, simulations find low temperature phases such as grain boundary states [71] or amorphous solids [72], but also perfect crystals distorted by density fluctuations [73]. Colloidal experiments reveal ground states with characteristics of a quenched hexatic glass [74], short range orientational order [75], or find a crystal-toglass transition [76]. There is no doubt that the structure of low temperature states as well as possible phase transitions to liquid-like phases is strongly depended on the range and strength of the quenched disorder. In the case of pinning disorder, especially when particles are pinned strongly, the freely moving rest of the ensemble experiences a weak confinement. To study the proximate effect of this confinement which might be responsible for the macroscopic phase behavior, we analyze the structure and dynamics of colloidal particles near pinning sites in a planar 2D-geometry.

\subsection{Experimental setup}

Our experimental system consists of a two-dimensional repulsive colloidal ensemble of superparamagnetic polystyrene beads confined to a glass substrate by gravity. The beads have a size of $d=4.5 \mu \mathrm{m}$, a mass density of $1.7 \mathrm{~kg} / \mathrm{dm}^{3}$ and a magnetic susceptibility of $\chi \approx 5.75 \cdot 10^{-11} \mathrm{Am}^{2} / \mathrm{T}$. The colloidal suspension is sealed within a cylindrical glass cell of $5 \mathrm{~mm}$ diameter in which the particles sediment to form a monolayer with density $n$ on the bottom glass plate. On the substrate, a small amount of them happens to pin due to van-der-Waals interaction or chemical reactions. The rest of the ensemble is able to move freely in two dimensions due to a weak repulsion with the glass substrate. The interaction within the ensemble is controlled by an external magnetic field $B$ perpendicular to the monolayer which induces a dipoledipole repulsion between the particles. The interaction strength can be quantified by the ratio of the mean magnetic energy between two particles with susceptibility $\chi$ and the thermal energy,

$$
\Gamma=\frac{\mu_{0}(\pi n)^{3 / 2}(\chi B)^{2}}{k_{B} T}
$$

which is proportional to the inverse system temperature $T^{-1}$. Regulating the magnetic field at constant density, we are able to test the phase behavior of the ensemble 


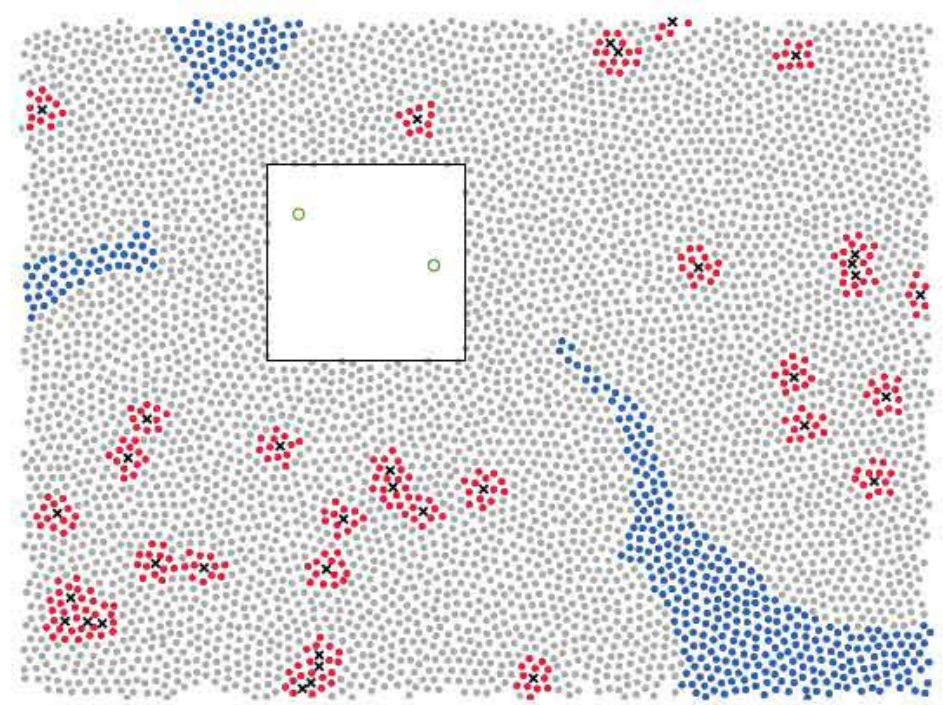

Fig. 11. Illustration of the subdivision of the colloidal ensemble. The field of view has the dimension $1158 \times 865 \mu \mathrm{m}^{2}$, consists of approx. 5540 particles with $a_{0} \approx 13.5 \mu \mathrm{m}$ and $\Gamma \approx 52$. Pinned sites are indicated with crosses, particles with $\Delta r<2 a_{0}$ are colored red, particles with $\Delta r>14 a_{0}$ blue. The inset shows $10000 \mathrm{~s}$ particle trajectories where pinned sites are indicated with green circles.

very precisely and with high resolution. Video microscopy allows us to track particles on large time scales and makes it possible to cover the phase space of the system. For this particular ensemble we observe the short time diffusion constant $D \approx$ $0.0295 \mu \mathrm{m}^{2} / \mathrm{s}$ and a Brownian time scale $\tau_{B}=(d / 2)^{2} / D \approx 172 \mathrm{~s}$.

\subsection{Results}

We are interested in the confining effect of the quenched disorder on particles in the vicinity of pinning sites, compared to the freely moving part of the system. We subdivide the free ensemble in two parts, one close $\left\{\mathbf{r}_{c}\right\}$ and one distant $\left\{\mathbf{r}_{d}\right\}$ from pinned particles $\left\{\mathbf{r}_{p}\right\}$, with

$$
\begin{gathered}
\left\{\mathbf{r}_{c}\right\}: \Delta r=\left|\mathbf{r}-\mathbf{r}_{p, i}\right|<2 a_{0} \\
\left\{\mathbf{r}_{d}\right\}: \Delta r=\left|\mathbf{r}-\mathbf{r}_{p, i}\right|>14 a_{0} \quad \forall \mathbf{r}_{p, i}
\end{gathered}
$$

where $a_{0}$ is the mean particle distance of the system. Figure 11 illustrates the subdivision for the ensemble at $\Gamma \approx 52$. At this interaction strength, the system is in the isotropic fluid phase with short range translational and orientational order. Phase transitions to the hexatic fluid and the solid phase with quasi-long-range and longrange orientational order occur at $\Gamma_{i} \approx 67$ and $\Gamma_{m} \approx 70$, respectively [77]. The inset of Fig. 11 shows particle trajectories revealing the trapping of the pinned sites.

To exhibit the local effect of the pinned particles on the dynamics and structure, we calculate the mean square displacement $(\Delta \mathbf{r})^{2}$ at short times and the pair correlation function $g(r)$ in the isotropic fluid $(\Gamma \approx 52)$ and the solid phase $(\Gamma \approx 108)$. The results are illustrated in Fig 12. In both the isotropic liquid and the solid, the mean square displacement (MSD) is decreased in the vicinity of the pinned particles, reflecting their confining character (on long timescales, this behaviour might 

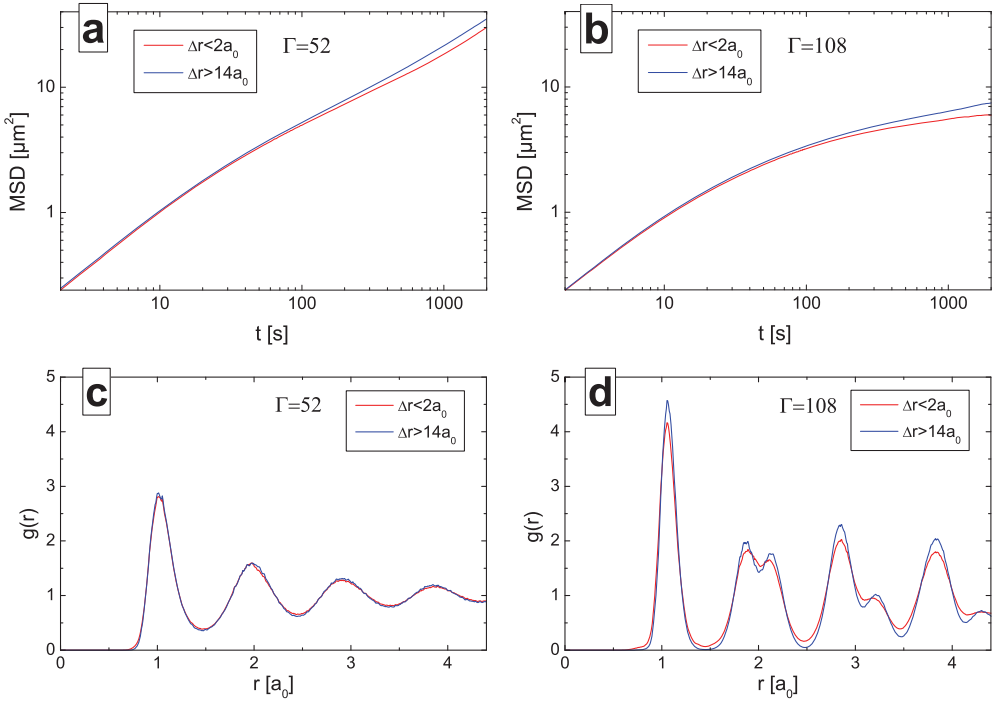

Fig. 12. Short time MSD $(t \leq 2000 \mathrm{~s})$ and $\mathrm{g}(\mathrm{r})$ in the isotropic fluid $(\mathrm{a}, \mathrm{c})$ and the solid $(b, d)$ for the different subsystem. The dynamics is clearly reduced close to pinned particles in the isotropic fluid and solid, visible by the drop of the MSD at the Brownian time scale $\tau_{B} \approx 172 \mathrm{~s}$. The structure is only affected in the solid where $\mathrm{g}(\mathrm{r})$ is weakened at distances corresponding to the underlying lattice and increased in between.

be reversed in the solid phase if the label, to which subsystem the particle belongs, changes each time it enters a new subsystem [77]). The different behaviour of the dynamics appears at the Brownian time scale $\tau_{B}$ and is of the same magnitude in both phases. The classification to which subsystem the particle belongs, is made only for the first time step and the MSD is calculated regardless whether particles leave the subsystem. For instance at $t=2000 \mathrm{~s}$, the MSD equals in the isotropic fluid $(\Delta \mathbf{r})^{2} \approx 30 \mu \mathrm{m}^{2}$ which corresponds to a distance of $\approx 0.4 a_{0}$. The structure behaves differently. While in the isotropic fluid $g(r)$ remains unaffected by pinning, it differs noticeably in the solid between close and distant particles. The probability to find particles in lattice related shells decreases while the probability that they appear at distances between shells increases. The lack of influence in the isotropic fluid is trivial since the system is disordered anyway and the effect of the pinned particles on the structure vanishes. In the solid, pinning sites induce a structural disorder caused by a possible incommensurability with the underlying lattice.

Melting in two dimensions is mediated by the dissociation of pairs of topological defects as dislocations and disclinations. To observe the effect of pinned sites on the general formation of those defects, we study the defect density $\rho$ during the melting process. The system is melted under equilibrium conditions from an initial crystalline state at $\Gamma \approx 120$ to a final isotropic state at $\Gamma \approx 5$. Figure 13 shows $\rho$ as a function of $\Gamma$. Whereas the overall defect density is increased compared to a system without pinning ( $\rho=2 \%$ at the melting temperature to the hexatic phase) it remains rather unaffected by the proximity to pinned sites such that the pinning density remains homogeneous.

In conclusion, we showed that the dynamics of the ensemble is inhibited in the vicinity of the pinned particles as a result of their local confining character. The structure is distorted only in the solid phase where pinning induces disorder due to its incommensurable character. Worth mentioning is that the effect on structure and dynamics is not directly correlated with the defect density distribution 


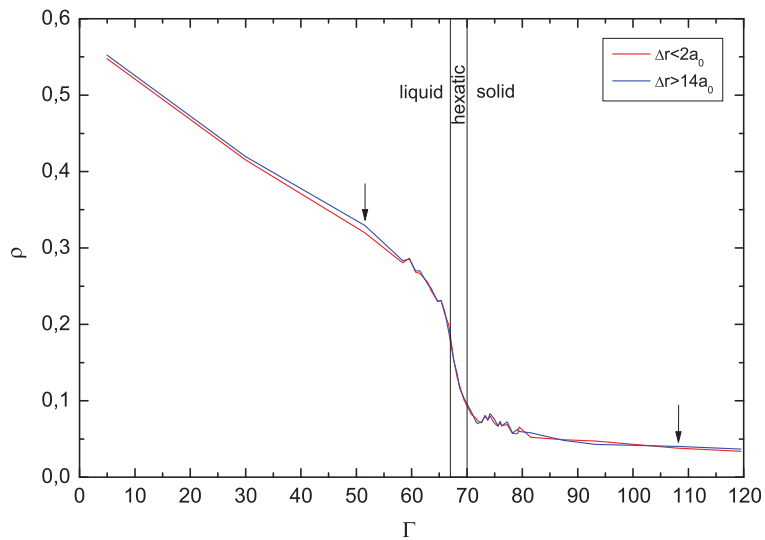

Fig. 13. Defect density $\rho$ as a function of $\Gamma$ for the two subsystems, defined as the number of particles with more or less than six neighbors divided by the total number of particles in the subsystem. The defect density remains rather homogeneous. The arrows mark the temperatures of data analysed in Fig. 12.

in the corresponding subsystem. This indicates that quenched disorder affects the system primarily rather on a macroscopic scale which, at least in the case of pinning, becomes evident in the structure and dynamics locally.

\section{Summary}

This paper has reviewed our recent studies on the effects of external fields on the structural and dynamical properties of colloidal systems in confined geometry: effects of uniform and uniaxial pressure, laser fields, shear due to moving boundaries, driving forces and randomly quenched disorder. The effect of compression and shear on the dynamics of colloidal particles in two-dimensional colloid crystals confined by corrugated walls has been investigated by computer simulations as well as the effect of external fields on phase diagrams in two-dimensional binary systems. In addition, the transport and diffusion properties of colloids in micro channels have been analyzed by Brownian Dynamics simulations. Experimental studies focused on the effect of quenched disorder on the local dynamics and structure in two dimensional systems.

We are grateful to S. Sengupta, the late I.K. Snook and N.B. Wilding for their valuable contributions to this work. D. Wilms acknowledges support from the Deutsche Forschungsgemeinschaft (DFG) under grant No TR6/C4 and from the Graduate School of Excellence Materials Science in Mainz. In earlier stages of this work A. Ricci and H.-Y. Chui have significantly contributed. P.N. and U.S. thank P. Leiderer, A. Erbe, and C. Kreuter for the experimental part of the joint collaborative work between project $\mathrm{C} 1$ and $\mathrm{C} 4$ of the $\mathrm{SFB}$ TR6, summarized in Sect. 4.2. We thank the NIC for computer time.

\section{References}

1. K. Franzrahe, P. Nielaba, S. Sengupta, Phys. Rev. E 82, 016112 (2010)

2. K. Franzrahe, P. Keim, G. Maret, P. Nielaba, S. Sengupta, Phys. Rev. E 78, 026106 (2008) 
3. K. Franzrahe, P. Nielaba, Phys. Rev. E 79, 051505 (2009)

4. K. Franzrahe, P. Nielaba, Phys. Rev. E 76, 061503 (2007)

5. F. Bürzle, P. Nielaba, Phys. Rev. E 76, 051112 (2007)

6. W. Strepp, S. Sengupta, P. Nielaba, Phys. Rev. E 63, 046106 (2001)

7. W. Strepp, S. Sengupta, P. Nielaba, Phys. Rev. E 66, 056109 (2002)

8. D.R. Nelson, in Phase Transitions and Critical Phenomena, edited by C. Domb and J.L. Lebowitz, Vol. 7 (Academic, London, 1983), p. 1

9. A. Ricci, P. Nielaba, S. Sengupta, K. Binder, Phys. Rev. E 74, 010404(R) (2006)

10. A. Ricci, P. Nielaba, S. Sengupta, K. Binder, Phys. Rev. E 75, 011405 (2007)

11. K. Binder, Y.-H. Chui, P. Nielaba, A. Ricci, S. Sengupta, in Nanophenomena at Surfaces: Fundamentals of Exotic Condensed Matter Properties, edited by M. Mihailov (Springer, Berlin, 2011), p. 1

12. D. Wilms, P. Virnau, I.K. Snook, K. Binder, Phys. Rev. B 86, 051404 (2012)

13. K. Bagchi, H.C. Andersen, W. Swope, Phys. Rev. E 53, 3794 (1996)

14. V.J. Emery, J.D. Axe, Phys. Rev. Lett. 40, 1507 (1978)

15. Y.-H. Chui, S. Sengupta, K. Binder, Europhys. Lett. 83, 58004 (2008)

16. Y.-H. Chui, S. Sengupta, I.K. Snook, K. Binder, J. Chem. Phys. 132, 074701 (2010)

17. Y.-H. Chui, S. Sengupta, I.K. Snook, K. Binder, Phys. Rev. E 81, 020403(R) (2010)

18. D. Wilms, N.B. Wilding, K. Binder, Phys. Rev. E 85, 056703 (2012)

19. M. Braun, Y.S. Kivshar, The Frenkel-Kontorova Model: Concepts, Methods, and Applications (Springer, Berlin, 2004)

20. W.C. Poon, P.N. Pusey, in Observation, Prediction, and Simulation of Phase Transitions in Complex Fluids, edited by M. Baus and L.F. Rull (Kluwer Acad. Publ., Dordrecht, 1995), p. 9

21. W. Goetze, J. Phys.: Cond. Mat. 11, A1 (1999)

22. K. Binder, W. Kob, Glassy Materials and Disorderd Solids. An Introduction to Their Statistical Mechanic, Revised Edition (World Scientific, Singapore, 2011)

23. HOOMD-blue webpage, http://codeblue.umich-edu/hoomd.blue

24. G.E. Murch, in Phase Transformations in Materials, edited by G. Kostorz (Wiley-VCH, Weinheim, 2001), p. 192

25. D. Wilms, P. Virnau, S. Sengupta, K. Binder, Phys. Rev. E 85, 061406 (2012)

26. D. Wilms, P. Virnau, K. Binder, Molecular Physics (in press)

27. B.N.J. Persson, E. Tosatti (eds.), Physics of Sliding Friction (Kluwer, Dordrecht, 1996)

28. Th. Bohlein, J. Mikhael, C. Bechinger, Nature Mater. 11, 126 (2012)

29. L. Lue, L.V. Woodcock, Mol. Phys. 96, 1435 (1999)

30. C.N. Likos, C.L. Henley, Phil. Mag. B 68, 85 (1993)

31. A. Chowdhury, B.J. Ackerson, Phys. Rev. Lett. 55, 833 (1985)

32. Q.H. Wei, C. Bechinger, D. Rudhardt, P. Leiderer, Phys. Rev. Lett. 81, 2606 (1998)

33. J. Chakrabarti, H.R. Krishnamurthy, A.K. Sood, S. Sengupta, Phys. Rev. Lett. 75, 2232 (1995)

34. P. Chaudhuri, C. Das, C. Dasgupta, H.R. Krishnamurthy, A.K. Sood, Phys. Rev. E 72 , $061404(2005)$

35. J. Chakrabarti, H.R. Krishnamurthy, A.K. Sood, Phys. Rev. Lett. 73, 2923 (1994)

36. E. Frey, D.R. Nelson, L. Radzihovsky, Phys. Rev. Lett. 83, 2977 (1999)

37. L. Radzihovsky, E. Frey, D.R. Nelson, Phys. Rev. E 63, 031503 (2001)

38. D. Wilms, S. Deutschländer, U. Siems, K. Franzrahe, P. Henseler, P. Keim, N. Schwierz, P. Virnau, K. Binder, G. Maret, P. Nielaba, J. Phys.: Cond. Mat. 24, 464119 (2012)

39. D. Helbing, P. Molnar, I. Farkas, K. Bolay, Environ. Plann. B Plann. Des. 28, 361 (2001)

40. T.M. Squires, S.R. Quake, Rev. Mod. Phys. 77, 977 (2005)

41. R. Roth, D. Gillespie, Phys. Rev. Lett. 95, 247801 (2005)

42. H. Löwen, J. Phys.: Condensed Matter 13, R415 (2005)

43. M. Rex, H. Löwen, C.N. Likos, Phys. Rev. E 72, 021404 (2005)

44. M. Rex, H. Löwen, Phys. Rev. E 75, 051402 (2007)

45. M. Rex, H. Löwen, Eur. Phys. J. E 26, 143 (2008)

46. J. Chakrabarti, J. Dzubiella, H. Löwen, Phys. Rev. E 70, 012401 (2004) 
47. J. Dzubiella, H. Löwen, J. Phys.: Condens. Matter 14, 9383 (2002)

48. J. Chakrabarti, J. Dzubiella, H. Löwen, EPL 61, 415 (2003)

49. L. Isa, R. Besseling, W.C.K. Poon, Phys. Rev. Lett. 98, 198305 (2007)

50. L. Isa, R. Besseling, A.N. Morozov, W.C.K. Poon, Phys. Rev. Lett. 102, 058302 (2009)

51. T. Vissers, A. van Blaaderen, A. Imhof, Phys. Rev. Lett. 106, 228303 (2011)

52. T. Vissers, A. Wysocki, M. Rex, H. Löwen, C.P. Royall, A. Imhof, A. van Blaaderen, Soft Matter 7, 2352 (2011)

53. P. Ballesta, G. Petekidis, L. Isa, W.C.K. Poon, R. Besseling, J. Rheology 56, 1005 (2012)

54. M.E. Leunissen, C.G. Christova, A.P. Hynninen, C.P. Royall, A.I. Campbell, A. Imhof, M. Dijkstra, R. v. Roij, A. v. Blaaderen, Nature 437, 235 (2005)

55. K.R. Sütterlin, A. Wysocki, A.V. Ivlev, C. Räth, H.M. Thomas, M. Rubin-Zuzic, W.J. Goedheer, V.E. Fortov, A.M. Lipaev, V.I. Molotkov, O.F. Petrov, G.E. Morfill, H. Löwen, Phys. Rev. Lett. 102, 085003 (2009)

56. B. Schmittmann, R.K.P. Zia, Phase Transition and Critical Phenomena, Vol. 17 (Academic, 1995)

57. N. Schwierz, P. Nielaba, Phys. Rev. E 82, 031401 (2010)

58. D.L. Ermak, J. Chem. Phys. 62, 4189 (1975)

59. R. Haghgooie, P.S. Doyle, Phys. Rev. E 70, 061408 (2004)

60. G. Piacente, F.M. Peeters, Phys. Rev. B 72, 205208 (2005)

61. M. Köppl, P. Henseler, A. Erbe, P. Nielaba, P. Leiderer, Phys. Rev. Lett. 97, 208302 (2006)

62. P. Henseler, A. Erbe, M. Köppl, P. Leiderer, P. Nielaba, Phys. Rev. E 81, 041402 (2010)

63. P.J. Steinhardt, D.R. Nelson, M. Ronchetti, Phys. Rev. B 28, 784 (1983)

64. U. Siems, Dissertation, U. Konstanz (in preparation)

65. B. Heinze, Diploma thesis, U Konstanz, 2013

66. Q.H. Wei, C. Bechinger, P. Leiderer, Science 287, 625 (2000)

67. U. Siems, C. Kreuter, A. Erbe, N. Schwierz, S. Sengupta, P. Leiderer, P. Nielaba, Nature - Sci. Rep. 2, 1015 (2012)

68. D.R. Nelson, Phys. Rev. B 27, 2902 (1983)

69. M.-C. Cha, H.A. Fertig, Phys. Rev. Lett. 74, 4867 (1995)

70. D. Carpentier, P. Le Doussal, Phys. Rev. Lett. 81, 1881 (1998)

71. A.C. Shi, A.J. Berlinsky, Phys. Rev. B 47, 652 (1993)

72. Z.-X. Cai, S. Sen, D.O. Welch, Phys. Rev. B 51, 15873 (1995)

73. S. Herrera-Velarde, H.H. von Grünberg, Soft Matter 5, 391 (2009)

74. R.E. Kusner, J.A. Mann, A.J. Dahm, Phys. Rev. B 49, 9190 (1994)

75. A. Pertsinidis, X.S. Ling, Phys. Rev. Lett. 100, 028303 (2008)

76. P. Yunker, Z. Zhang, A.G. Yodh, Phys. Rev. Lett. 104, 015701 (2010)

77. S. Deutschländer, T. Horn, H. Löwen, G. Maret, P. Keim, Phys. Rev. Lett. 111, 098301 (2013) 